\title{
Development and evaluation of coenzyme Q10 loaded solid lipid nanoparticle hydrogel for enhanced dermal delivery
}

\author{
EMRAH KORKMAZ* \\ EVREN H. GOKCE \\ OZGEN OZER
}

Department of Pharmaceutical Technology Faculty of Pharmacy, University of Ege Izmir, 35100, Turkey

\begin{abstract}
Coenzyme Q10 (Q10) loaded solid lipid nanoparticles (SLN) were prepared by the high speed homogenization method and incorporated into Carbopol 974P hydrogels. Compritol 888 ATO (C888) was employed as the lipid base; Poloxamer 188 (P188) and Tween 80 (Tw80) were used as surfactant and co-surfactant. Optimum particle size with narrow distribution was obtained as $152.2 \mathrm{~nm}$ for blank and $142.4 \mathrm{~nm}$ for Q10 loaded SLNs. The overall charge of loaded SLNs was $-13.7 \pm 1.3 \mathrm{mV}$. Q10 entrapment efficiency was $89 \%$ and the production yield was $94 \%$. Transmission electron microscopy analysis provided evidence of colloidal size, spherical shape while differential scanning calorimetry analysis confirmed recrystallization of the lipid after the preparation of SLNs. Trolox equivalent antioxidant capacity (TEAC) analysis has shown that antioxidant potential of Q10 can be protected in SLNs. Rheological characteristics demonstrated that the SLN incorporating gels were shear thinning and the mechanical strength of the gels was suitable for topical application. Diffusion studies from rat abdominal skin revealed that the delivery of Q10 was doubled in SLN incorporating gels, approximately $40 \mu \mathrm{g} \mathrm{cm}^{-2}$, in comparison with gels prepared with only Q10 (not incorporated in SLNs). As a result, it can be stated that Q10-SLN loaded gels can be successful delivery systems for carrying Q10 efficiently into the skin without losing its antioxidant properties.
\end{abstract}

Keywords: antioxidant, coenzyme Q10, skin delivery, hydrogel, solid lipid nanoparticles

Skin is the protective organ of the body with an area around $2 \mathrm{~m}^{2}$. The hazardous potential of toxins, pathogens and UV radiation are avoided by the protection that skin supplies (1). UV radiation is the main source of energy that initiates reactive oxygen

\footnotetext{
* Correspondence; e-mail: evrenhomangokce@gmail.com
} 
species (ROS). Formation of ROS leads to oxidative damage in DNA, proteins and lipids. The skin has an enzymatic (superoxide dismutase, catalase, and glutathione peroxidase) and non-enzymatic (antioxidant) protection mechanism against these damages; however, excess ROS production overcomes this protection and causes several diseases such as cancer $(2,3)$. Hence, effective delivery of an antioxidant into skin in required levels is an important challenge. There are two main steps limiting the effective delivery antioxidants, oxidation of the active substance and carrying the active substance to the viable epidermis.

Antioxidants are defined as substances that, when present in a low concentration compared to that of oxidizable substrates, significantly delay or inhibit oxidation of that substance (4). In general, antioxidants act by the chain breaking reaction, by reducing the concentration of reactive oxygen species, by scavenging initiating radicals and by chelating transition metal catalysts (5). Coenzyme Q10 (Q10) is the only lipid soluble endogenous antioxidant. It supports ATP generation during aerobic cellular respiration in the electron transport chain in the mitochondria and inhibits cell membrane peroxidation in reduced form $(6,7)$. However, it is very difficult to prepare a formulation that helps Q10 delivery to deeper layers of the skin due to the low aqueous solubility of Q10 $(\log P>10)$ and due to the barrier function of stratum corneum (8).

In the second half of the nineties there was an increasing interest in investigating solid lipid nanoparticles (SLNs) for dermal application. SLNs have many advantages, especially for dermal applications such as occlusion, modulation of drug release and penetration into the skin (9). Since they are made from solid lipids, chemically unstable lipid soluble compounds that are sensitive to oxidation can be successfully encapsulated in the SLNs $(8,10)$. Several molecules were incorporated into lipid nanoparticles, e.g., ascorbyl palmitate, resveratrol, retinol and retinyl palmitate, vitamin $\mathrm{E}$ and vitamin $\mathrm{E}$ acetate $(8,11-14)$. It has been shown that systemic uptake of active substances (e.g., isotretinoin) through skins could be avoided and SLN could increase the accumulative uptake of the active material in the skin (15). SLNs are produced as liquid dispersions and their incorporation into hydrogels or emulsions helps enhance the retention time in the applied area and patient compliance $(16,17)$.

Hydrogels are generally formed from carbomers that have the ability to absorb water, get hydrated and swell by neutralization with a base. Carbomers have been mainly used in liquid or semi-solid pharmaceutical formulations in order to modify their flow characteristics $(17,18)$.

The aim of this work was to carry an efficient amount of Q10 to epidermis via SLNs without being oxidized. This study was therefore designed to prepare Q10 loaded SLNs and incorporate them into carbomer based hydrogels. The physicochemical characterization of SLNs, Trolox equivalent antioxidant capacity (TEAC) analysis and rheological characterization of hydrogels were conducted. Ex vivo diffusion studies were used to determine the accumulated amount of Q10 in rat skin.

\section{EXPERIMENTAL}

\section{Materials}

Compritol ${ }^{\circledR} 888$ ATO (Glyceryl behenate) and Labrasol ${ }^{\circledR}$ were obtained from Gattefossée (France). Poloxamer ${ }^{\circledR} 188$ (Pluronic F68) was donated by BASF (Germany). Tween ${ }^{\circledR}$ 
E. Korkmaz et al.: Development and evaluation of coenzyme Q10 loaded solid lipid nanoparticle hydrogel for enhanced dermal delivery, Acta Pharm. 63 (2013) 517-529.

80 (Polysorbate 80) and Q10 were obtained from Sigma Aldrich (Italy). Carbopol 974P was donated by Lubrizol (Germany). All HPLC reagents and chloroform were purchased from Sigma Aldrich (Italy). The other chemicals were obtained from Merck. All filters were purchased from Sartorius (Germany).

\section{Preparation of SLNS}

Q10 loaded SLNs were prepared by the high shear homogenization method (19) using two different probes, $8 \mathrm{G}$ and 10G, at different concentrations of ingredients (Table I). C888 was employed as the lipid base; Poloxamer 188 (P188) and Tween 80 (Tw 80) were used as surfactant and co-surfactant, respectively. As reported in our previous study (14), the lipid phase Compritol 888 ATO (C888) and Q10- were mixed and heated to $85^{\circ} \mathrm{C}$. At the same time, the aqueous phase consisting of P188 and Tw 80 in $12.5 \mathrm{~mL}$ bidistilled water was heated to the same temperature. The aqueous phase was mixed with the lipid phase by Ultra-Turrax (IKA-T25, Germany) at $24000 \mathrm{rpm}$. The pre-emulsion produced was dispersed in $12.5 \mathrm{~mL}$ bidistilled cold water $\left(4^{\circ} \mathrm{C}\right)$ and kept in the freezer $\left(-20^{\circ} \mathrm{C}\right)$ for 10 minutes to help solidification of nanoparticles. Blank SLNs were prepared in a similar way, without addition of Q10 (Table I).

\section{Measurement of particle size and polydispersity index}

The particle size (PS) and polydispersity index (PI) of SLNs were measured by Photon Correlation Spectroscopy (Malvern Zetasizer-NanoZS, UK) at $25{ }^{\circ} \mathrm{C}$. The formulations were diluted with bidistilled and filtered $(0.45 \mu \mathrm{m})$ water before the experiment $(n$ $=6$ ). Each sample was measured in triplicate.

\section{Measurement of zeta potential}

The zeta potential (ZP) of SLN dispersions was measured at $25{ }^{\circ} \mathrm{C}$ under an electrical field of $40 \mathrm{~V} \mathrm{~cm}^{-1}$ (Malvern Zetasizer-NanoZS, UK). The measurements were conducted in triplicate.

Table I. Content of ingredients in the aqueous dispersion of solid nanoparticles

\begin{tabular}{ccccc}
\hline \multirow{2}{*}{ Formulation } & \multicolumn{4}{c}{ Content $(\%, m / V)$} \\
\cline { 2 - 5 } & C888 & P188 & Tw80 & Q10 \\
\hline F1-8G & 0.4 & 0.2 & 0.1 & - \\
F2-8G & 0.8 & 0.4 & 0.4 & - \\
F3-8G & 1.2 & 0.6 & 0.3 & - \\
F1-10G & 0.4 & 0.2 & 0.1 & - \\
F2-10G & 0.8 & 0.4 & 0.4 & - \\
F3-10G & 1.2 & 0.6 & 0.3 & 0.02 \\
F3-Q10-10G & 1.2 & 0.6 & 0.3 & \\
\hline
\end{tabular}


E. Korkmaz et al.: Development and evaluation of coenzyme Q10 loaded solid lipid nanoparticle hydrogel for enhanced dermal delivery, Acta Pharm. 63 (2013) 517-529.

\section{Drug entrapment efficiency}

One $\mathrm{mL}$ of SLN dispersion was placed in a dialysis bag (cut-off $25 \mathrm{kDa}$ ). The bags were centrifuged for $1 \mathrm{~h}$ at $14000 \mathrm{rpm}$ in a medium consisting of $5 \%$ Labrasol $^{\circledR}(8)$. The amount of Q10 in $5 \%$ Labrasol solution was then analyzed by a validated HPLC method and the quantity of free drug was calculated. The encapsulated amount of Q10 was calculated by subtracting the free amount of Q10 from the total amount in the dispersion. Each batch was evaluated three times. The following equation was used to calculate the entrapment efficiency $(E E, \%)$, where $W_{\mathrm{i}}$ is the amount of initial drug and $W_{\mathrm{f}}$ is the amount of free drug.

The HPLC system (Agilent Series 1100, Italy) consisted of a C18 reverse phase column (ACE 5-C18 $250 \mathrm{~mm} \times 4.6 \mathrm{~mm}$ ). The mobile phase was a mixture of methanol/ $n$-hexan 65:35 $(V / V)$. The flow rate and UV wavelength were set at $1 \mathrm{~mL} \mathrm{~min}^{-1}$ and 275 nm, respectively (20).

\section{Determination of production yield}

Nanoparticle suspensions were frozen at $-80{ }^{\circ} \mathrm{C}$ for $24 \mathrm{~h}$. Freeze-drying was performed at a pressure of $7 \mathrm{kPa}$ for $24 \mathrm{~h}$ at $-45^{\circ} \mathrm{C}$ (Christ Alpha 1-2 LD Freeze Dryer). The ratio of total theoretical amount to lyophilized powder was calculated.

\section{Transmission electron microscopy (TEM) analysis}

A transmission electron microscope (CM12 Philips, the Netherlands) was used for the morphological examination of SLNs. $2 \%(w / V)$ phosphotungistic acid was used to stain the samples and after staining they were placed on copper grids for viewing.

\section{Differential scanning calorimetry (DSC) analysis}

Q10, C888, blank and loaded SLN formulations were analyzed. The samples to be tested were weighed between $6-8 \mathrm{mg}$, sealed in aluminum pans $(50 \mu \mathrm{L})$ and placed in a differential scanning calorimeter (DSC 8000 Perkin Elmer). DSC analysis was conducted under nitrogen flow $\left(20 \mathrm{~mL} \mathrm{~min}{ }^{-1}\right)$ in a temperature range from $30-300{ }^{\circ} \mathrm{C}$ with a heat flow of $20{ }^{\circ} \mathrm{C} \mathrm{min}-1$.

\section{TEAC experiments}

Antioxidant activity was measured using the 2,2'-azino-bis(3-ethylbenzothiazoline-6-sulfonic acid) (ABTS) method (21).

Potassium persulfate $\left(2.45 \mathrm{~mol} \mathrm{~L}^{-1}\right)$ was mixed with ABTS $(7 \mathrm{~mol} \mathrm{~L}-1)$ in order to produce an ABTS+ radical. This radical was left in a dark room at $30{ }^{\circ} \mathrm{C}$ for 2 days to reach stable absorbance at $734 \mathrm{~nm}$.

One $\mathrm{mL}$ of this solution was placed into a cuvette and its absorbance was detected as AABTS.+. Then Q10 in $5 \%$ Labrasol and SLN formulations were added into the solu-

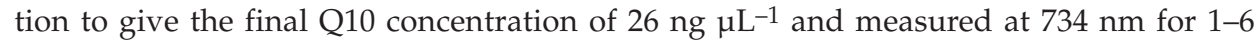
min after the initial mixing. All measurements were carried out in triplicate. Inhibition percentages were calculated as follows: 
E. Korkmaz et al.: Development and evaluation of coenzyme Q10 loaded solid lipid nanoparticle hydrogel for enhanced dermal delivery, Acta Pharm. 63 (2013) 517-529.

$$
\text { Inhibition \% } \left.=\text { (AABTS. }_{+}-\text {A6.min }\right) \times 100 / \text { AABTS. }_{+}
$$

where AABTS..$_{+}$is the absorbance of $\mathrm{ABTS}_{+}$at $734 \mathrm{~nm}(0.700 \pm 0.02)$ and A6.min is the 6-min absorbance after the addition of the sample to the $\mathrm{ABTS}_{+}$.

The standard curve of Trolox was obtained using a Trolox standard solution at various concentrations $\left(2.5-15 \mathrm{mmol} \mathrm{L}^{-1}\right)$ in a buffer. Absorbance of the sample was compared to that of the standard curve and the antioxidant property was expressed as mol $\mathrm{L}^{-1}$ Trolox equivalent/mg.

\section{Preparation of gels}

Small portions of Carbopol 974P $(0.5 \%, \mathrm{~m} / \mathrm{m})$ were added into a SLN solution under continuous stirring (1500 rpm) at room temperature for 3-5 h. After 24 hours, the gels were neutralized with sodium hydroxide $\left(c=2 \mathrm{~mol} \mathrm{~L}^{-1}\right)$ to give a gel matrix with a $\mathrm{pH}$ value of 5.4. The prepared gels were centrifuged at $3000 \mathrm{rpm}$ for $15 \mathrm{~min}$ to avoid bubbles and kept in the refrigerator for one night before evaluation. Blank gels were prepared in the same way without addition of SLNs.

\section{Mechanical properties of gels}

Textural profile analysis (TPA) was performed using a software-controlled penetrometer (TA-TX Plus, Stable Micro Systems, Godalming, UK) equipped with a $500 \mathrm{~g}$ load cell in the texture profile analysis mode $(17,18)$. An analytical probe was compressed twice into the formulations in glass vials to a defined depth $(1.5 \mathrm{~mm})$ and at a defined rate $\left(8 \mathrm{~mm} \mathrm{~s}^{-1}\right)$, allowing a delay period $(15 \mathrm{~s})$ between the end of the first and beginning of the second compression. Mechanical parameters (hardness, compressibility, adhesiveness, cohesiveness, and elasticity) were derived from the resultant force-time curve. Each experiment was conducted on six different samples.

\section{Rheological studies}

All formulations were characterized rheologically using a Haake Mars rheometer (Thermo Fisher, Karlsruhe, Germany). Continuous shear analysis of formulations was performed at 25 and $37^{\circ} \mathrm{C}( \pm 0.1)$, in the flow mode, and in conjunction with cone-plate geometry (diameter $35 \mathrm{~mm}$ ) with a gap of $0.105 \mathrm{~mm}$. Formulations were placed on the lower plate and allowed to equilibrate for at least 1 minute prior to analysis.

Oscillatory analysis of each formulation was performed at $25^{\circ} \mathrm{C}$ after determination of its linear viscoelastic region, where the stress was directly proportional to the strain and the storage modulus remained constant. Frequency sweep analysis was performed over the frequency range of $0.1-10.0 \mathrm{~Hz}$ following application of constant stress at $2 \mathrm{~Pa}$. Storage modulus $\left(G^{\prime}\right)$, loss modulus $\left(G^{\prime \prime}\right)$ and dynamic viscosity $(\eta)$ were determined. In each case, the dynamic rheological properties of at least six replicates were examined.

\section{Ex vivo diffusion studies}

Male, 200-300 g weighing Wistar-albino rat's abdominal skin was excised immediately after the animal was sacrificed, and purified from the surrounding tissue. The skin was placed in a Franz diffusion cell and $600 \mu \mathrm{L}$ of the formulations (Q10 solution in $5 \%$ 
Labrasol, Q10 gel, Q10-SLN and Q10 loaded SLN gel) were evaluated. The receptor phase consisted of $5 \%$ Labrasol solution at $37^{\circ} \mathrm{C}$ and sink conditions were taken into consideration. The formulations to be tested were kept in contact with the tissue for $24 \mathrm{~h}$ and, at certain time intervals, the Q10 amount was determined in $300 \mu \mathrm{L}$ samples taken from the receptor phase. After $24 \mathrm{~h}$, skin samples were taken, the excess formulation was removed, and skin surface was cleaned with dry paper. Tissue samples were cut into small pieces and Q10 accumulated in the skin was extracted with the aid of a horizontal shaker in $2 \mathrm{~mL}$ of methanol/n-hexan (65:35) for $24 \mathrm{~h}$.

\section{Statistical analysis}

Statistical analysis was conducted by ANOVA followed by Tukey's test for comparisons between groups. 0.05 was taken as the $p$ value, to indicate statistical significance ( $p$ $<0.05)$.

\section{RESULTS AND DISCUSSION}

Physical properties of nano-systems might affect the in vivo behavior. In our previous study, it was reported that particle size and zeta potential have important roles in the dermal penetration properties of lipid nanoparticles (10). The data in terms of PS and PI of SLNs are given in Table II. As can be seen from Table II, an increment in the lipid base concentration caused an increment in particle size. The effect of C 888 concentration on the PS values was more obvious with probe 8G. The variability of PS was enhanced with probe 8G. Therefore the formulations prepared with Probe 10G were more uniform and the inter-formulation variability was lower.

Increment of lipids in the system increased the PS detected. PI is a very important parameter to understand the uniformity of the particle size dimension; values below 0.5 indicate the uniformity of the system. If the system is not uniform, aggregation may occur. The PI was small enough to state that the formulations prepared with the bigger probe $10 \mathrm{G}$ were homogenous in terms of dimension. Nano-sized particles have some

Table II. The particle size and polydispersity index of formulations ${ }^{a}$

\begin{tabular}{ccc}
\hline Formulation & PS $(\mathrm{nm})$ & PI \\
\hline F1-8G & $232.95 \pm 27.90$ & $0.502 \pm 0.07$ \\
F2-8G & $268.00 \pm 17.94$ & $0.402 \pm 0.01$ \\
F3-8G & $336.40 \pm 82.71$ & $0.405 \pm 0.04$ \\
F1-10G & $137.40 \pm 21.40$ & $0.364 \pm 0.03$ \\
F2-10G & $152.20 \pm 14.04$ & $0.351 \pm 0.04$ \\
F3-10G & $164.10 \pm 9.35$ & $0.294 \pm 0.05$ \\
F3-Q10-10G & $142.40 \pm 17.90$ & $0.272 \pm 0.03$ \\
\hline
\end{tabular}

a Mean \pm SD 
advantages in overcoming the stratum corneum barrier and penetrating into deeper layers of the skin. Nano-size can act in favor of the interaction of solid lipids of the formulation with lipids of the stratum corneum and passing through the pores in the skin (10, 19). The smallest and homogenous dispersion was obtained with F3. The production yield was $94 \pm 1 \%$.

Addition of Q10 into the lipid of F3 helped to form smaller particles. This behavior could be attributed to the polymorphism of Q10 and the lipid. Since Q10 is a highly lipophilic substance, it is likely that Q10 was solubilized in C888, which might affect the recrystallization process or the activity of surfactants.

DSC analysis was performed to evaluate the crystallization. It can be seen from Fig. 1 that the peak of Q10 at $54{ }^{\circ} \mathrm{C}$ disappeared indicating the solubility in the lipid phase and that there was a slight change in the melting point peak of C888 in SLNs (from 77 to $74^{\circ} \mathrm{C}$ ). This change might be responsible for the smaller particle size detected. As the lipid melted earlier, a homogenous pre-emulsion could be formed with smaller droplets. In a previous study, Q10 was found to be dissolved in the lipid matrix (cetyl palmitate), similarly to the results of our study. After cooling, the absence of the melting peak of Q10 was attributed to the formation of a super-cooled melted state, amorphous or molecular dispersed state of Q10 in the mixture (15). It was seen in another study that the height of the endotherm in the thermogram of SLN containing Q10 was less than that in SLN without Q10, suggesting loss of crystallinity of the lipids and Q10 after incorporation into SLN (11).

Q10 addition reduced the overall charge of the SLNs from -(18.6 \pm 1.2$)$ to $-(13.7 \pm$ 1.3) $\mathrm{mV}$, as it was also seen in our previous studies conducted with cyclosporine A (22). The drug entrapment efficiency of Q10 was determined as $89 \%$ by a validated HPLC method. This high encapsulation amount was expected due to very high lipophilicity of Q10 $(\log P>10)$.

Colloid dimension and spherical shape of SLNs are supported by TEM images (Fig. 2).

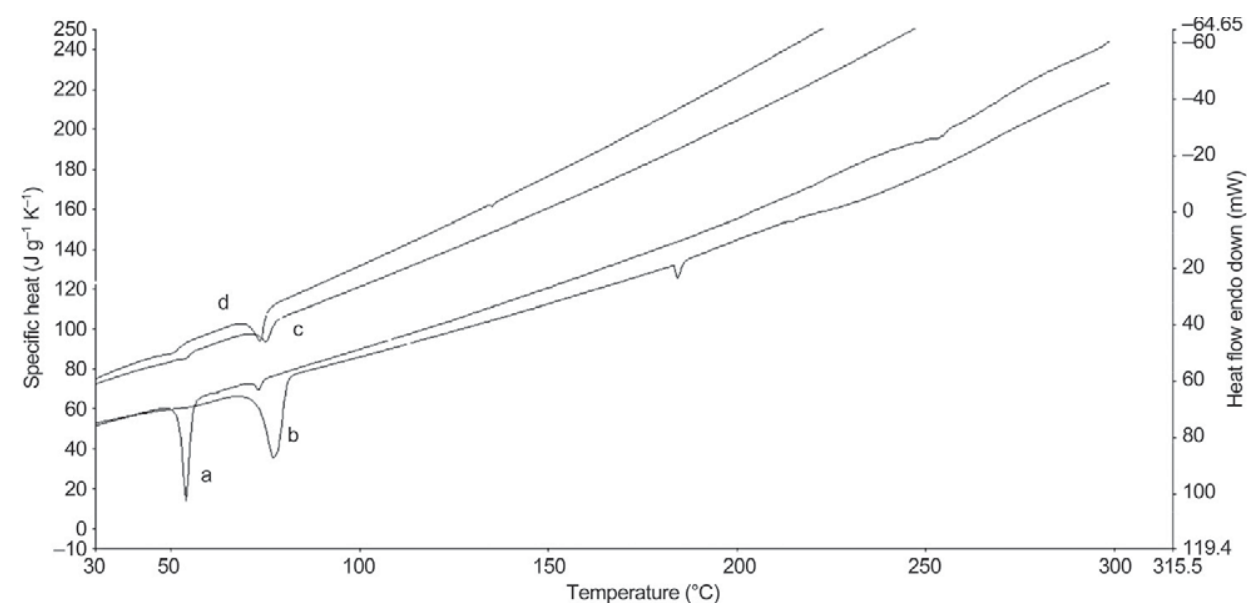

Fig. 1. Thermograms of: a) Q10, b) C888, c) blank and d) Q10 loaded SLNs obtained with DSC. 


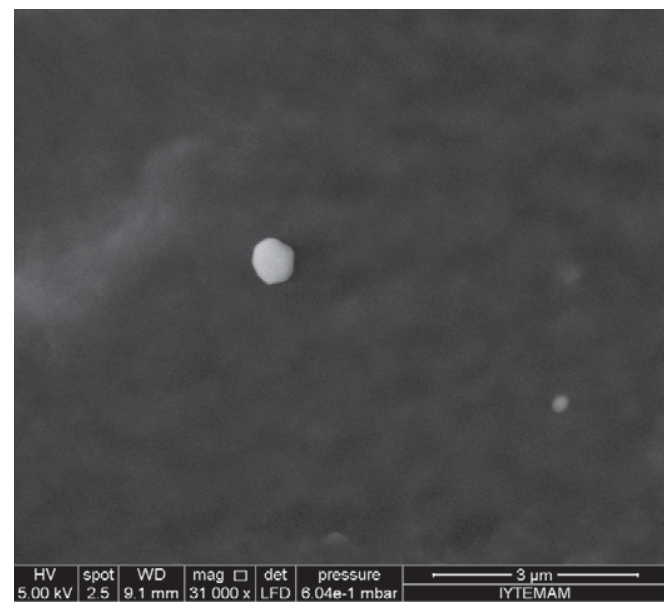

Fig. 2. TEM image of Q10 loaded SLN.

It is known that the structure of SLNs protects labile drugs from degradation or oxidation by encapsulation $(8-11,17)$. TEAC experiments showed that the encapsulation in SLNs was successful in protecting Q10 from oxidation. Q10 in $5 \%$ Labrasol solution and Q10 loaded SLNs displayed TEAC around 11 and $0.28 \mathrm{mM} / \mathrm{L}$, respectively. This result indicated that ABTS radical was not able to interact with Q10 whilst it was protected by a lipid matrix.

Q10 loaded SLNs were successfully prepared but their application onto the skin to deliver the required amount of Q10 into deeper layers was very important. SLN incorporating gels were formed to increase the retention time on the applied area. Three different formulations were prepared; blank gels, Q10 incorporating gels and Q10 loaded SLN incorporating gels. Texture profile analysis was conducted to understand the mechanical properties of the gels. As can be seen from Table III, the addition of SLNs did not change the mechanical properties of gels ( $p>0.05$ for all parameters).

It is known that the parameters such as hardness, compressibility, adhesiveness, cohesiveness affect the performance of topical semisolid formulations, e.g., ease of removal from the container, ease of application to the surface and retention of the product at the site of application. Compressibility is the force required to deform the samples during compression. Hardness and compressibility describe the stress/work required to remove the sample from the container and to subsequently spread over the site of application. These characteristics quantify sample deformation under compression. Gel hardness and compressibility should be low to allow the gel to be easily removed from the container and spread onto the bio-surface $(23,24)$.

It was reported in a study of Jones et al. that sodium carboxymethylcellulose at a concentration of $6 \%$ had compressibility of $1.87 \pm 0.34 \mathrm{~N} \mathrm{~mm}$ (24). Similarly, in a study of Bonacucina et al., $1.240 \pm 0.46 \mathrm{~N} \mathrm{~mm}$ was reported to be suitable for topical administration. (25). Our results are in accord with these previous studies.

In this study, elastic modulus $\left(G^{\prime}\right)$, viscous modulus $\left(G^{\prime \prime}\right)$ and viscosity were monitored to define the gel structure. The elastic (or storage) modulus represents elastic stor- 
E. Korkmaz et al.: Development and evaluation of coenzyme Q10 loaded solid lipid nanoparticle hydrogel for enhanced dermal delivery, Acta Pharm. 63 (2013) 517-529.

Table III. Mechanical properties of gels obtained by TPA ${ }^{a}$

\begin{tabular}{lccccc}
\hline Formulation & $\begin{array}{c}\text { Hardness } \\
(\mathrm{N})\end{array}$ & $\begin{array}{c}\text { Compressibility } \\
(\mathrm{N} \mathrm{mm})\end{array}$ & $\begin{array}{c}\text { Adhesiveness } \\
(\mathrm{N} \mathrm{mm})\end{array}$ & Cohesiveness & Elasticity \\
\hline Blank gel & $0.178 \pm 0.008$ & $1.121 \pm 0.101$ & $0.833 \pm 0.073$ & $0.976 \pm 0.836$ & $0.595 \pm 0.040$ \\
Q10-Gel & $0.191 \pm 0.002$ & $1.155 \pm 0.663$ & $0.757 \pm 0.581$ & $0.973 \pm 0.001$ & $0.540 \pm 0.325$ \\
Q10SLN-Gel & $0.195 \pm 0.005$ & $1.479 \pm 0.323$ & $1.080 \pm 0.268$ & $0.967 \pm 0.026$ & $0.706 \pm 0.087$ \\
\hline
\end{tabular}

a Mean \pm SD.

age of energy and it is a measure of a well-structured material. The viscous (or loss) modulus represents loss of viscous energy and it will be large when the sample is viscous (26). In the presence of a gel structure, $G^{\prime}$ and $G^{\prime \prime}$ are frequency independent whilst for concentrated solutions there is a frequency dependence of $G^{\prime}$ and $G^{\prime \prime}$ (27). A definition of Burchard and Ross-Murphy (28) is that a gel shows a plateau in the real part of the complex modulus extending over an appreciable window of frequencies. Briefly, $\mathrm{G}^{\prime}$ mo-

a)

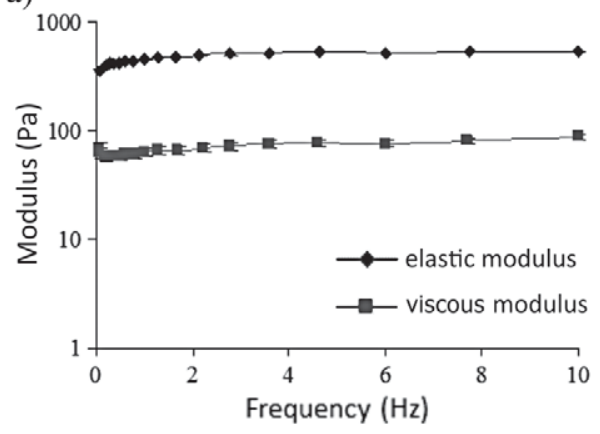

b)

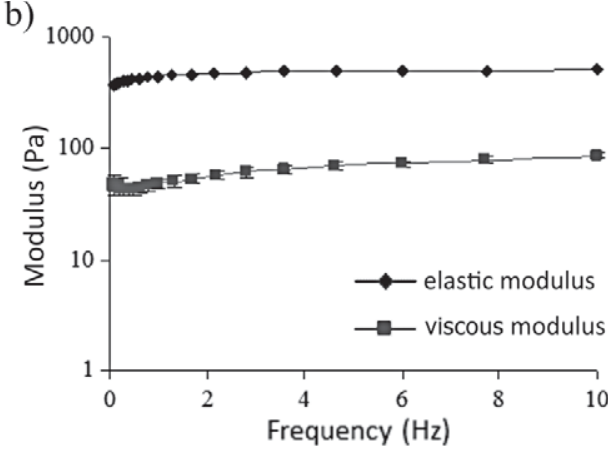

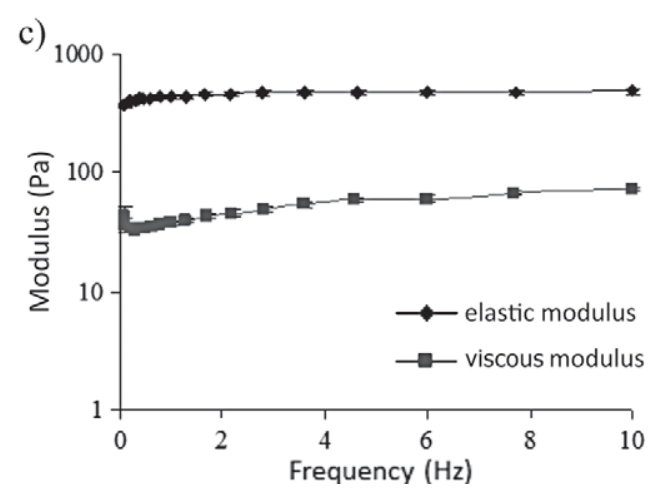

Fig. 3. Oscillation graphics of: a) blank gel, b) Q10 gel and c) Q10 loaded SLN incorporating gel at $25^{\circ} \mathrm{C}$. 
E. Korkmaz et al.: Development and evaluation of coenzyme Q10 loaded solid lipid nanoparticle hydrogel for enhanced dermal delivery, Acta Pharm. 63 (2013) 517-529.

dulus had to be greater than $G^{\prime \prime}$ modulus in the entire frequency range examined and both moduli had to be frequency independent.

As can be seen from Fig. 3, the oscillation graphics indicated that the formulations prepared were of gel characters since $G^{\prime}$ was greater than $G^{\prime \prime}$. Incorporation of SLNs into gels did not affect gel behavior.

Viscosity profiles (Fig. 4) showed that the gels started flowing when shear stress was increased. This kind of behavior indicated a shear-thinning system for all formulations. Similarly, in a previous study Carbopol hydrogels incorporating liposomes and cyclodextrin have shown shear-thinning behavior (29).

Farboud et al. (11) evaluated the release of Q10 loaded SLN oil/water creams through cellulose nitrate membranes. It was reported in their study that the percentage of released Q10 from simple cream was higher than for SLN and SLN cream in the first hour, but after $7 \mathrm{~h}$ SLN and SLN cream released almost all of the Q10 content whereas simple cream released only $40 \%$. However, in our study in which rat abdominal skin was used, there was no diffusion of Q10 through the receptor phase during $24 \mathrm{~h}$ of observation. Therefore the amount of Q10 accumulated in the skin could be determined.

a)

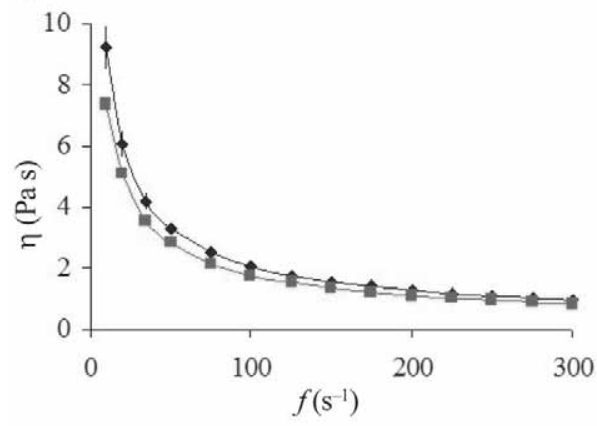

b)

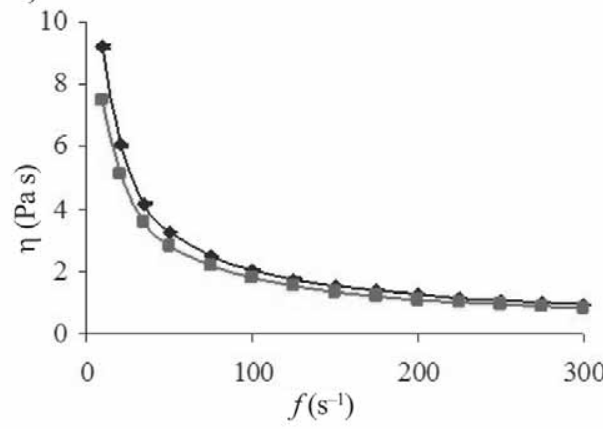

c)

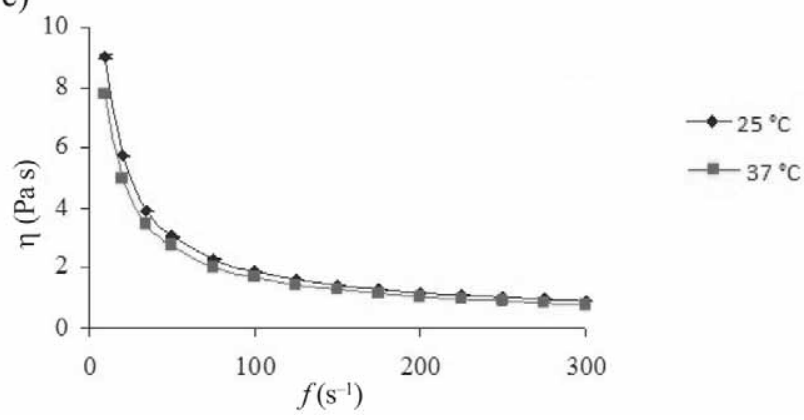

Fig. 4. Viscosity vs. shear rate profiles of: a) blank gel, b) Q10 gel and c) Q10 loaded SLN incorporating gel at room and body temperature. 
Fig. 5. The amount of Q10 determined from the rat abdominal skin tissue.

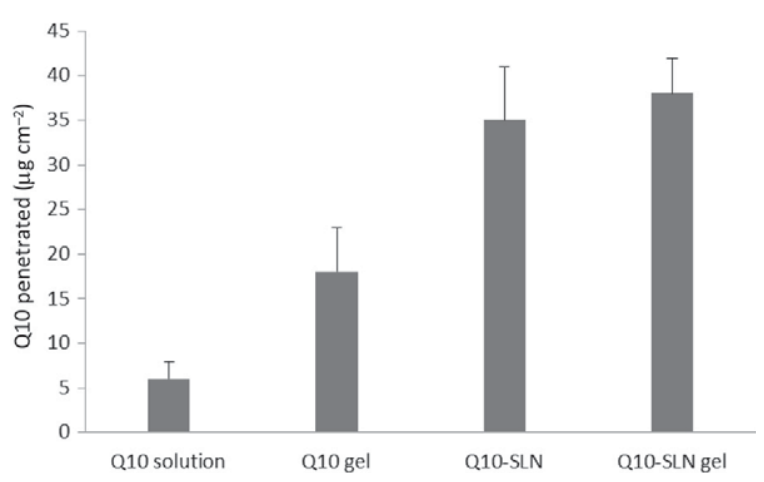

As can be seen from Fig. 5, the penetration of Q10 into the skin was increased by its encapsulation in the SLN formulation. The small size and lipophilicity of the particles helped the delivery of Q10 into the skin. The delivery of Q10 was almost doubled in SLN and SLN incorporating gels compared to the gel formulation prepared with solely Q10 (not incorporated in SLNs). It is known that small size ensures close contact with the stratum corneum and can increase the amount of drug absorbed by the skin (12). Zhaou et al. (30) reported that Q10 loaded lipid nanocapsules accumulated in both epidermis and dermis. Since oxidative stress affects several pathways, the delivery of Q10 into the skin is particularly important (31). The gel without SLNs also acted in favor of Q10 penetration. It was supposed that as the residence time of Q10 was prolonged, the penetration of Q10 could be elevated.

\section{CONCLUSIONS}

Q10 loaded SLNs could be successfully prepared by the high speed homogenization method with high drug loading and production yield. The antioxidant activity of Q10 could be protected from environmental conditions in the SLN matrix. SLN incorporation into hydrogels did not make dramatic changes in the gel's mechanical and rheological characteristics. The formulation obtained was suitable for topical, especially dermal, applications. Even the prolongation of Q10 presence in the application area increased its skin penetration. In addition, the nano-size obtained by a lipid matrix of SLN helped to establish closer contact with stratum corneum and increased the amount of Q10 absorbed by the skin in comparison with other formulations. As a result, it could be concluded that Q10-SLN incorporating gels can be regarded successful delivery systems for delivering Q10 efficiently into the skin without losing its antioxidant properties.

Acknowledgement. - This study was supported by the University of Ege Scientific Research Foundation, Project No. 09/ECZ/030. 
E. Korkmaz et al.: Development and evaluation of coenzyme Q10 loaded solid lipid nanoparticle hydrogel for enhanced dermal delivery, Acta Pharm. 63 (2013) 517-529.

\section{REFERENCES}

1. U. Hoppe, J. Bergemann, W. Diembeck, J. Ennen, S. Gohla, I. Harris, J. Jacob, J. Kielholz, W. Mei, D. Pollet, D. Schachtschabel, G. Sauerman, V. Schreiner, F. Stäb and F. Steckel, Coenzyme Q10, a cutaneous antioxidant and energizer, Biofactors 9 (1999) 371-378.

2. P. D. Forbes, Photocarcinogenesis: an overview, J. Invest. Dermatol. 77 (1981) 139-143; DOI: 10. 1111/1523-1747.ep12479351.

3. M. Afzal, B. Matsugo, K. Aoyama and T. Takeuchi, Method to overcome photoreaction, a serious drawback to the use of dichlorofluorescin in evaluation of reactive oxygen species, Biochem. Biophys. Res. Commun. 304 (2003) 619-624; DOI: 10.1016/S0006-291X(03)00641-7.

4. C. R. Murthy, K. V. Rama Rao, G. Bai and M. D. Norenberg, Ammonia-induced production of free radicals in primary cultures of rat astrocytes, J. Neurosci. Res. 66 (2001) 282-288; DOI: 10. 1002 /jnr.1222.

5. S. Kumar, Free radicals and antioxidants: human and food system, Adv. Appl. Sci. Res. 2 (2011) 129-135.

6. G. R. Lenaz, Fato, G. Formiggini and M. L. Genova, The role of Coenzyme Q in mitochondrial electron transport, Mitochondrion 7 (2007) Suppl:S8-S33; DOI: 10.1016/j.mito.2007.03.009.

7. K. Muta-Takada. T. Terada, H. Yamanishi, Y. Ashida, S. Inomata, T. Nishiyama and S. Amano, Coenzyme Q10 protects against oxidative stress-induced cell death and enhances the synthesis of basement membrane components in dermal and epidermal cells, Biofactors 35 (2009) 435-441; DOI: 10.1002 /biof.56.

8. V. Teeranachaideekul, E. B. Souto, V. B. Junyaprasert and R. H. Müller, Encapsulation of ascorbyl palmitate in nanostructured lipid carriers NLC - effects of formulation parameters on physicochemical stability, Int. J. Pharm. 340 (2007) 198-206; DOI: 10.1016/j.ijpharm.2007.03.022.

9. E. B. Souto. S. A. Wissing, C. M. Barbosa and R. H. Müller, Development of a controlled release formulation based on SLN and NLC for topical clotrimazole delivery, Int. J. Pharm. 278 (2004) 71-77; DOI: 10.1016/j.ijpharm.2004.02.032.

10. E. H. Gokce, E. Korkmaz, E. Dellera, G. Sandri, M. C. Bonferoni and O. Ozer, Resveratrol-loaded solid lipid nanoparticles versus nanostructured lipid carriers: evaluation of antioxidant potential for dermal applications, Int. J. Nanomed. 7 (2012) 1841-1850; DOI: 10.2147\%2FIJN.S29710.

11. E. S. Farboud. S. A. Nasrollahi and Z. Tabbakhi, Novel formulation and evaluation of a Q10-loaded solid lipid nanoparticle cream: in vitro and in vivo studies, Int. J. Nanomed. 6 (2011) 611617; DOI: 10.2147\%2FIJN.S16815.

12. K. Teskac and J. Kristl, The evidence for solid lipid nanoparticles mediated cell uptake of resveratrol, Int. J. Pharm. 390 (2010) 61-69; DOI: 10.1016/j.ijpharm.2009.10.011.

13. V. Jenning and S. H. Gohla, Encapsulation of retinoids in solid lipid nanoparticles (SLN), J. Microencapsul. 18 (2001) 149-158; DOI: 10.1080/02652040010000361.

14. A. Dingler, R. P. Blum, H. Niehus, R. H. Muller and S. Gohla, Solid lipid nanoparticles (SLN/ Lipopearls)-a pharmaceutical and cosmetic carrier for the application of vitamin $\mathrm{E}$ in dermal products, J. Microencapsul. 16 (1999) 751-767; DOI: 10575627.

15. V. Teeranachaideekul, E. B. Souto, V. B. Junyaprasert and R. H. Muller, Cetyl palmitate based NLC for topical delivery of Coenzyme Q(10) - development, physicochemical characterization and in vitro release studies, Eur. J. Pharm. Biopharm. 67 (2007) 141-148. DOI: 10.1016/j.ejpb.2007. 01.015.

16. J. Liu, W. Hu, H. Chen, Q. Ni, H. Xu and X. Yang, Isotretinoin-loaded solid lipid nanoparticles with skin targeting for topical delivery, Int. J. Pharm. 328 (2007) 191-195. DOI: 10.1016/j. ijpharm.2006.08.007.

17. S. Y. Karavana, E. H. Gökçe, S. Rençber, S. Ozbal, C. Pekçetin, P. Güneri and G. Ertan, A new approach to the treatment of recurrent aphthous stomatitis with bioadhesive gels containing cy- 
E. Korkmaz et al.: Development and evaluation of coenzyme Q10 loaded solid lipid nanoparticle hydrogel for enhanced dermal delivery, Acta Pharm. 63 (2013) 517-529.

closporine A solid lipid nanoparticles: in vivo/in vitro examinations, Int. J. Nanomed. 7 (2012) 5693-5704; DOI: $10.2147 \% 2 F I J N . S 36883$.

18. D. S. Jones, M. L. Bruschi, O. de Freitas, M. P. Gremião, E. H. Lara and G. P. Andrews, Rheological, mechanical and mucoadhesive properties of thermoresponsive, bioadhesive binary mixtures composed of poloxamer 407 and carbopol 974P designed as platforms for implantable drug delivery systems for use in the oral cavity, Int. J. Pharm. 372 (2009) 49-58; DOI: 10.1016/j. ijpharm.2009.01.006.

19. E. H. Gokce, E. Korkmaz, S. Tuncay-Tanriverdi, E. Dellera, G. Sandri, M. C. Bonferoni and O. Ozer, A comparative evaluation of coenzyme Q10-loaded liposomes and solid lipid nanoparticles as dermal antioxidant carriers, Int. J. Nanomed. 7 (2012) 5109-5117; DOI: 10.2147/IJN.S 34921.

20. J. Karpinska. B. Mikoluć, R. Motkowski and J. Piotrowska-Jastrzebska, HPLC method for simultaneous determination of retinol, alpha-tocopherol and coenzyme Q10 in human plasma, J. Pharm. Biomed. Anal. 18 (2006) 232-236; DOI: 10.1016/j.jpba.2006.03.037.

21. R. Re, N. Pellegrini and A. Proteggente, Antioxidant activity applying an improved ABTS radical cation decolorization assay, Free Radic. Biol. Med. 26 (1999) 1231-1237; DOI: 10.1016/S08915849(98)00315-3.

22. E. H. Gokce, G. Sandri, M. C. Bonferoni, S. Rossi, F. Ferrari, T. Güneri and C. Caramella, Cyclosporine A loaded SLNs: evaluation of cellular uptake and corneal cytotoxicity, Int. J. Pharm. 364 (2008) 76-86; DOI: 10.1016/j.ijpharm.2008.07.028.

23. D. S. Jones, A. D. Woolfson and J. Djokic, Texture profile analysis of bioadhesive polymeric semisolids: mechanical characterization and investigation of interactions between formulation components, J. Appl. Polym. Sci. 61 (1996) 2229-2234; DOI: 10.1002/(SICI)1097-4628(19960919) 61:12<2229::AID-APP24>3.0.CO;2-0.

24. D. S. Jones, A. D. Woolfson and A. F. Brown, Textural, viscoelastic and mucoadhesive properties of pharmaceutical gels composed of cellulose polymers, Int. J. Pharm. 151 (1997) 223-233; DOI: 10.1016/S0378-5173(97)04904-1.

25. G. Bonacucina, M. Cepsi, M. Misici-Falzi and G. F. Palmieri, Rheological, adhesive and release characterisation of semisolid carbopol/tetraglycol systems, Int. J. Pharm. 307 (2005) 29-14; DOI: 10.1016/j.ijpharm.2005.09.034.

26. S. Tamburic and D. Q. M. Craig, Rheological evaluation of polyacrylic acid hydrogels, Pharm. Sci. 1 (1995) 107-109; DOI: 10.1111/j.2042-7158.1995.tb00403.x.

27. J. Carlfors, K. Edsman, R. Petersson and K. Jörnving, Rheological evaluation of Gelrite ${ }^{\circledR}$ in situ gels for ophthalmic use, Eur. J. Pharm. Sci. 6 (1998) 113-119; DOI: 10.1016/S0928-0987(97)00074-2.

28. W. Burchard and S. B. Ross-Murphy, Polymers and Gels, Elsevier Science Publishers, London 1990, pp. 15-23.

29. L. Boulmedarat, J. L. Grossiord, E. Fattal and A. Bochot, Influence of methyl- $\beta$-cyclodextrin and liposomes on rheological properties of Carbopol ${ }^{\circledR}$ 974P NF gels, Int. J. Pharm. 254 (2003) 59-64; DOI: 10.1016/S0378-5173(02)00683-X.

30. H. Zhou, Y. Yue, G. Liu, Y. Li, J. Zhang, Z. Yan and M. Duan, Characterisation and skin distribution of lecithin-based coenzyme Q10-loaded lipid nanocapsules, Nanoscale Res. Lett. 20 (2010) 1561-1569; DOI: 10.1007/s11671-010-9677-z.

31. D. R. Bickers and M. Athar, Oxidative stress in the pathogenesis of skin disease, J. Invest. Dermatol. 126 (2006) 2565-2575; DOI: 10.1038/sj.jid.5700340. 